\title{
Formulación de políticas públicas sobre el cáncer de mama en América Latina
}

\author{
M. C. González-Robledo, ${ }^{1}$ L. M. González-Robledo ${ }^{2}$ y G. Nigenda ${ }^{1}$
}

Forma de citar

González-Robledo MC, González-Robledo, LM, Nigenda G. Formulación de políticas públicas sobre el cáncer de mama en América Latina. Rev Panam Salud Publica. 2013;33(3):183-9.

RESUMEN Objetivo. Conocer el proceso de formulación de política pública sobre la atención del cáncer de mama en cinco países de América Latina.

Métodos. Estudio evaluativo exploratorio realizado en Argentina, Brasil, Colombia, México y Venezuela en 2010. La muestra de países seleccionados fue de conveniencia. Se realizaron 65 entrevistas semiestructuradas a funcionarios gubernamentales, académicos y representantes de gremios profesionales y de organizaciones de la sociedad civil. Se realizó un análisis de contenido para fuentes secundarias. Se integraron fuentes de información, datos e informantes para el análisis mediante el método de triangulación.

Resultados. Los países con mayor avance en la formulación de políticas públicas sobre el cáncer de mama son Brasil y México. Argentina, Colombia y Venezuela, pese a no tener una politica definida, disponen de programas y acciones para su atención. Se distinguen dos perspectivas de desarrollo para estas políticas públicas: una con amplia participación de los sectores gubernamental y no gubernamental, y otra más restringida a la participación exclusiva de autoridades gubernamentales.

Conclusiones. Los resultados traducen importantes diferencias entre países en la formulación de políticas públicas sobre el cáncer de mama en la Región y destacan el mayor avance en aquellos países donde se han desarrollado a través de procesos de participación incluyente.

Palabras clave Políticas públicas de salud; neoplasias de la mama; América Latina.

El cáncer de mama es una enfermedad que está consolidándose como un problema prioritario de salud pública en América Latina (1). Se estima que en 2020 en el mundo habrá cerca de dos millones de casos nuevos, de los cuales $76 \%$ residirán en países de ingresos medios y bajos, y de estos, 20\% en América Latina (2), donde cada año se diagnostican

\footnotetext{
Instituto Nacional de Salud Pública, Cuernavaca, Morelos, México. La correspondencia se debe dirigir a María Cecilia González-Robledo. Correo electrónico: cecilia.gonzalez@insp.mx

2 Universidad Autónoma del Estado de Morelos, Facultad de Medicina, Cuernavaca, Morelos, México.
}

114900 casos nuevos y mueren a causa de la enfermedad 37000 mujeres (3).

Según estudios publicados, en la Región de las Américas se ha producido un incremento importante de la incidencia y la mortalidad de este cáncer en los últimos 30 años. La tasa de incidencia aumentó de 18 a 35\% entre 1980 y 2008, y la de mortalidad, $18 \%$ entre 2002 y 2008, con gran variabilidad regional (mayores tasas en países del cono sur, como Uruguay y Argentina) (4). Considerando el envejecimiento de la población en la Región (en 2050 se habrá triplicado la fracción de ancianos, que habrá pasado de 8 a $24 \%$ de la población) $(5,6)$, se espera que la incidencia y la prevalencia de esta enfermedad aumenten, dado que está asociada al envejecimiento (7). Una de sus consecuencias será el aumento de la carga económica de la enfermedad, que afectará tanto a los sistemas de salud como a las familias (8).

Debido al reto que para los gobiernos y los sistemas de salud impone la atención del cáncer de mama en la Región, es importante conocer de qué manera se están preparando los diferentes países para afrontar este desafío. Es fundamental idear soluciones integrales que respondan con equidad, calidad y protección financiera a la atención de 
las necesidades y expectativas de la población, ya que el pronóstico clínico de las pacientes mejora y la mortalidad disminuye cuando la organización de los procesos de atención es adecuada, es decir, cuando existen programas debidamente estructurados y dotados de los recursos apropiados para prestar dicha atención (9-11).

Las políticas públicas se definen como "las decisiones válidamente adoptadas por un organismo público (nacional, estatal o local) en respuesta a una situación o problema relevante, que afecte seriamente a la población u organismos de su jurisdicción" (12). En este proceso decisorio, la formulación de políticas públicas implica tomar decisiones sobre qué hacer, para qué, cómo, con qué y con quiénes (13), mediante el análisis de los comportamientos, las circunstancias y las decisiones de actores clave y sobre la base de la información provista por personas que desempeñan en él papeles $\mathrm{y}$ funciones relevantes (14).

El presente artículo documenta los procesos de formulación de políticas públicas en cinco países de América Latina: Argentina, Brasil, Colombia, México y Venezuela, para identificar buenas prácticas y casos exitosos, así como dificultades que han afrontado al intentar dar respuesta a este problema de salud pública de la Región.

\section{MATERIALES Y MÉTODOS}

Los resultados presentados en este documento forman parte de una evaluación más amplia denominada "Políticas y programas gubernamentales de atención al cáncer de mama en América Latina: México, Colombia, Venezuela, Brasil y Argentina", realizada por el Instituto Nacional de Salud Pública (INSP) de México con el apoyo financiero de la American Cancer Society. Su objetivo principal se centró en analizar las políticas y los programas gubernamentales para la atención del cáncer de mama en cinco países de América Latina, con el fin de identificar avances y retos en la formulación e implementación de políticas gubernamentales para su atención. Para los fines del presente artículo, se presentan los resultados del análisis del proceso de formulación de políticas públicas para la atención del cáncer de mama en los cinco países de la muestra evaluada.

La base conceptual del análisis de la información se basó en dos modelos.
Primero, el propuesto por Hogwood y Gunn (15), referente a las etapas de la política pública que, en este caso particular, se centró en tres subetapas: la definición del problema, el proceso de la toma de decisiones y la legitimación de la política a través de la acción normativa. El análisis se complementó con el segundo modelo, el de los determinantes de política pública de Dye (16), que se basa en el estudio de sus procesos y contenidos. Se prestó especial atención a la información brindada por los diversos informantes respecto al papel desempeñado por múltiples actores en este proceso. Los entrevistados aportaron conocimientos, percepciones y experiencias de acuerdo con sus propios intereses, ideologías y recursos materiales.

En el estudio se emplearon métodos de recolección y análisis cualitativos, y la información se recabó en fuentes de información primaria y secundaria. La información primaria se obtuvo entre julio y noviembre de 2010. La muestra para el estudio se extrajo de manera intencional en dos etapas. En la primera, se seleccionaron los países de acuerdo con los siguientes criterios: 1) diversidad geográfica, 2) tasas de prevalencia, incidencia y mortalidad del cáncer de mama, 3) evidencia previa sobre el desarrollo de acciones gubernamentales y no gubernamentales para la atención del cáncer de mama, 4) interés propio de la organización que brindó el apoyo finan-

ciero al proyecto, y 5) interés de los actores locales para participar en el estudio. En la segunda etapa se seleccionaron los actores clave y se obtuvo información documental a través de la técnica de la bola de nieve (17).

La información primaria se consiguió realizando entrevistas semiestructuradas a funcionarios gubernamentales, académicos y a representantes de gremios profesionales y de organizaciones de la sociedad civil (cuadro 1). El criterio principal para definir a los actores clave fue la posibilidad de obtener respuestas basadas en la experiencia y el conocimiento sustantivo a las preguntas incluidas en las guías de entrevista. En total se hicieron 65 entrevistas, cuya cifra final se determinó a través del proceso de saturación teórica (18). Se utilizaron guías de entrevista, lo que facilitó la exploración sistemática de los temas abordados. Las entrevistas se grabaron en un medio digital, previo consentimiento informado por parte del informante. Se realizó su trascripción y se guardaron en archivos de Word. Posteriormente, los datos se codificaron teniendo en cuenta las categorías definidas, que se organizaron en matrices sistematizadas y se clasificaron según componente, tema e informante.

Las fuentes de información secundaria incluyeron documentos oficiales que contenían información sobre normatividad y directrices de políticas relacionadas con el tema estudiado (cuadro 2). Su

\section{CUADRO 1. Fuentes de información primaria por país en América Latina, 2010}

\begin{tabular}{lcccccc}
\hline \multicolumn{1}{c}{ Informante } & México & Colombia & Venezuela & Brasil & Argentina & Total \\
\hline Sector gubernamental & 7 & 5 & 3 & 3 & 5 & 23 \\
Sociedad civil organizada & 3 & 5 & 8 & 3 & 3 & 22 \\
Gremios profesionales & 0 & 1 & 3 & 3 & 1 & 8 \\
Otros actores & 4 & 2 & 4 & 1 & 1 & 12 \\
Total & 14 & 13 & 18 & 10 & 10 & 65 \\
\hline
\end{tabular}

CUADRO 2. Fuentes de información secundaria por país en América Latina, 2010

\begin{tabular}{lccccc}
\hline \multicolumn{1}{c}{ Tipo de documento } & México & Colombia & Venezuela & Brasil & Argentina \\
\hline Salud de la mujer & $\checkmark$ & $\checkmark$ & $\checkmark$ & $\checkmark$ & $\checkmark$ \\
Salud sexual y reproductiva & $\checkmark$ & $\checkmark$ & $\checkmark$ & $\checkmark$ & $\checkmark$ \\
Políticas para cáncer de mama & $\checkmark$ & $\checkmark$ & - & $\checkmark$ & $\checkmark$ \\
$\begin{array}{l}\text { Programas para la atención/control del cáncer } \\
\quad \text { de mama }\end{array}$ & $\checkmark$ & & $\checkmark$ & $\checkmark$ & $\checkmark$ \\
Consensos para tratamiento del cáncer de mama & $\checkmark$ & $\checkmark$ & $\checkmark$ & $\checkmark$ & $\checkmark$ \\
Estadísticas de cáncer de mama (por encuestas & $\checkmark$ & $\checkmark$ & $\checkmark$ & $\checkmark$ & - \\
$\quad$ nacionales/regionales) & & & & &
\end{tabular}

$\checkmark$ Existencia física o electrónica de documentos de soporte.

- No se encontraron documentos de soporte. 
propósito fue identificar conceptos clave o fases del proceso de construcción de la política pública, particularmente de la acción normativa. Las categorías bajo las cuales se presentan los resultados son las siguientes (19):

1. Definición del problema: es el proceso mediante el cual la sociedad identifica necesidades y carencias que deben resolverse. Para ello se requiere disponer de información sólida, suficiente y oportuna. Esa categoría también se conoce como reconocimiento del problema.

2. Posicionamiento del tema en la agenda política: es el procedimiento mediante el cual una situación recibe la denominación de problema público, cuando un determinado grupo con influencia y capacidad de movilizar actores de poder considera que la situación actual no es aceptable y se necesita la intervención derivada de una política pública para remediarla.

3. Proceso de toma de decisiones o etapa de formalización: se basa en la construcción y la selección de opciones para resolver el problema identificado.

4. Legitimación de la política: es el proceso por el cual se establecen acuerdos gubernamentales, que corresponden a las acciones legislativas y normativas para resolver el problema identificado.
El análisis de la información se realizó a través del método inductivo, usando elementos básicos de la teoría fundamentada propuesta por Glaser y Strauss (20). A la información obtenida de fuentes secundarias se aplicó el método de análisis de contenido (21). El análisis de la información buscó integrar las diferentes fuentes y métodos de recolección de información mediante la técnica de la triangulación (22).

Para este estudio se aplicaron los criterios éticos establecidos en la declaración de Helsinki (23). El proyecto original de investigación fue aprobado por las comisiones de Investigación, Ética y Bioseguridad del Instituto Nacional de Salud Pública de México.

\section{RESULTADOS}

En todos los países se pudieron identificar las cuatro fases que conforman el diseño de una política pública mencionadas anteriormente.

\section{Definición del problema}

En los cinco países estudiados la han realizado principalmente -aunque no de manera exclusiva - actores no gubernamentales y académicos, llevando a cabo en los últimos 10 años investigaciones y estudios epidemiológicos para entender, entre otras cosas, su magnitud y distribución. En el cuadro 3 se presenta información estadística del cáncer de mama por país, obtenida de fuentes primarias y secundarias (30-35).

2. Posicionamiento del tema en la agenda política

Se observaron características comunes en la Región: 1) el problema del cáncer de mama ha estado ligado al del cáncer en general ( $y$, en particular, al del cáncer cervicouterino), así como a políticas de salud de la mujer; 2) su reconocimiento como problema de salud pública se debe en gran medida al incremento sostenido de las tasas de incidencia y mortalidad de la enfermedad en los últimos 30 años, y 3) en países como Argentina, Colombia y México la incorporación del tema a la agenda gubernamental se ha producido en los últimos cinco o seis años gracias a las presiones de grupos de interés, particularmente de organizaciones de la sociedad civil de mujeres con cáncer.

\section{Proceso de toma de decisiones}

En la Región se ha desarrollado de dos maneras. Primera, a través de procesos incluyentes, es decir, con participación activa de sectores del gobierno y de la sociedad civil, como en Brasil y Mé-

\section{CUADRO 3. Información estadística del cáncer de mama en cinco países de América Latina}

\begin{tabular}{|c|c|c|}
\hline País & Datos & Tipo de fuente (referencias) \\
\hline Argentina & $\begin{array}{l}\text { El cáncer de mama es la primera causa de muerte por enfermedad maligna en mujeres (20,3\%). El } \\
\text { país tiene la segunda tasa de mortalidad en la Región de las Américas ( } 21,8 \text { por } 100.000 \text { mujeres). } \\
\text { Se estima que anualmente se diagnostican alrededor de } 17000 \text { casos nuevos (2008). De éstos se } \\
\text { considera que } 58 \% \text { se diagnosticará en mujeres mayores de } 60 \text { años, } 18 \% \text {, de } 50 \text { a } 60 \text { años, } 18 \% \text {, } \\
\text { de } 40 \text { a } 50 \text { años, y } 6 \% \text {, en menores de } 40 \text { años. }\end{array}$ & Gubernamental (24) y no gubernamental (25) \\
\hline Brasil & $\begin{array}{l}\text { El cáncer de mama es el tumor maligno más frecuente y la primera causa de muerte por } \\
\text { enfermedad maligna en mujeres. Se estimaron casi } 50000 \text { casos nuevos en } 2010 \text {, que representan } \\
\text { el } 27 \% \text { de todos los casos nuevos de cáncer en mujeres en el país. Su tasa nacional de incidencia } \\
\text { ajustada por edad fue de } 49 \text { por } 100000 \text {. Sin embargo, se observaron importantes diferencias } \\
\text { regionales, mayores en el sur del país. El número de muertes por esta causa en } 2008 \text { fue de } 12 \\
573 \text {, lo que equivale al } 14 \% \text { de las muertes por cáncer en las mujeres. }\end{array}$ & No gubernamental (26) y gubernamental (27) \\
\hline Venezuela & $\begin{array}{l}\text { El cáncer de mama en Venezuela es la segunda causa de muerte por cáncer en mujeres (15,6\%) y } \\
\text { ocupa el noveno lugar por mortalidad general en mujeres (3\%). Cada día se diagnostican } 9 \text { casos } \\
\text { nuevos. El número de casos estimados en } 2008 \text { fue de } 3564 \text { y la tasa de incidencia estandarizada } \\
\text { fue de } 27,4 \text { por } 100000 \text { mujeres. }\end{array}$ & No gubernamental $(28,29)$ \\
\hline Colombia & $\begin{array}{l}\text { Se estima que la tasa de incidencia del cáncer de mama en Colombia es de } 30,3 \text { por } 100000 \text { y la } \\
\text { de mortalidad, de } 11,0 \text { por } 100 \text { 000. Este tipo de cáncer se encuentra entre las primeras } 20 \text { causas } \\
\text { de muerte en el } 70 \% \text { del país. Las tasas de mortalidad han aumentado } 13,2 \% \text { entre } 1960 \text { y } 2009 \text {. }\end{array}$ & $\begin{array}{l}\text { No gubernamental }(30,31) \\
\text { y gubernamental }(32)\end{array}$ \\
\hline México & $\begin{array}{l}\text { El cáncer de mama es la segunda causa de muerte por cáncer en mujeres mayores de } 25 \text { años. } \\
\text { La tasa de incidencia en } 2008 \text { fue de } 27,2 \text { por } 100000 \text { y la de mortalidad, de } 10,1 \text { por } 100 \text { 000, y } \\
\text { ha mostrado una tendencia incremental entre } 1980 \text { y 2005), de } 3,6 \% \text { en } 18 \text { años. Desde } 2005 \text {, la } \\
\text { mortalidad por este cáncer supera la mortalidad por cáncer cervicouterino. En } 2010 \text {, de los casos } \\
\text { confirmados, más del } 50 \% \text { fueron diagnosticados en estadios avanzados (III y IV). }\end{array}$ & $\begin{array}{l}\text { No gubernamental }(33,34) \\
\text { y gubernamental }(35)\end{array}$ \\
\hline
\end{tabular}


xico. Colombia está iniciando un proceso de participación intersectorial. Segunda, mediante una participación más restringida y circunscrita en el sector gubernamental (Argentina y Venezuela). En el cuadro 3 se presentan las aportaciones de los diferentes actores en la definición de la política. El cuadro 4 muestra el papel desempeñado por cada uno de los actores en la toma de decisiones.

En países como Brasil, Colombia y México se pone de manifiesto una interacción entre legisladores, funcionarios públicos, grupos de interés y la población general a través de la cual se han alcanzado algunos acuerdos. No obstante esta interacción, se necesitan mayores espacios de participación. En el caso concreto de México destacan la actualización de la norma técnica para la atención del cáncer de mama, con la cual, gracias a un proceso de negociación, se logró bajar la edad para realizar la primera mamografía de control de los 50 a los 40 años de edad. En Brasil, por norma, las organizaciones de la sociedad civil participan en las decisiones de los consejos municipales, estatales y nacionales, lo que les permite contribuir al diseño, formulación e implementación de las políticas. Sin embargo, algunos actores de la sociedad civil recomiendan reforzar los espacios de participación. En Argentina,

CUADRO 4. Participación de los actores en la toma de decisiones para la formulación de política pública sobre el cáncer de mama en cinco países de América Latina, 2010

\begin{tabular}{|c|c|}
\hline Tipo de actor & Tipo de participación \\
\hline Legislativo & $\begin{array}{l}\text { - Elaboración y aprobación de iniciativas de ley para la atención integral del } \\
\text { cáncer de mama en el ámbito nacional. } \\
\text { - Regulación de las acciones contra el cáncer. } \\
\text { - Aprobación de los presupuestos para la atención de la enfermedad. }\end{array}$ \\
\hline Gubernamental & $\begin{array}{l}\text { - Define, instrumenta, supervisa y evalúa las estrategias y los contenidos } \\
\text { técnicos de la política o programa sobre cáncer de mama (presentación } \\
\text { de proyectos de normatividad, es decir, un conjunto de regulaciones, } \\
\text { lineamientos teóricos y técnicos y recomendaciones dirigidos a garantizar } \\
\text { que las intervenciones propuestas sean adecuadas para la población y que } \\
\text { estén de acuerdo con el marco legal general del país). } \\
\text { - Tiene a su cargo la ejecución del financiamiento de los servicios que se } \\
\text { prestan a la población. Esto se realiza a través de diversos mecanismos: } \\
\text { 1) de manera directa (Brasil, México), 2) a través del pago por terceros } \\
\text { (sistemas de aseguramiento en salud, como en Colombia y Venezuela), } \\
\text { 3) otros (sistemas mixtos, como en Argentina). }\end{array}$ \\
\hline \multirow[t]{2}{*}{$\begin{array}{l}\text { Organizaciones de la } \\
\text { sociedad civil (gremios } \\
\text { profesionales, académicos, } \\
\text { organizaciones de mujeres) }\end{array}$} & $\begin{array}{l}\text { - En la prestación de servicios. Define las intervenciones (acciones o } \\
\text { paquetes de servicios) que serán prestados por el sector público y los de la } \\
\text { seguridad social. } \\
\text { - Se han constituido como grupos de interés para incluir el tema en la agenda } \\
\text { gubernamental (abogacía). }\end{array}$ \\
\hline & $\begin{array}{l}\text { - Participan con la generación de información relevante (conocimiento } \\
\text { técnico) para la toma de decisiones (investigación, evaluación, guías y } \\
\text { protocolos de atención), que han logrado comprobar la importancia del } \\
\text { problema en los ámbitos de la salud pública y económico (carga de la } \\
\text { enfermedad, gasto público y de bolsillo, etc.). }\end{array}$ \\
\hline
\end{tabular}

algunos actores entrevistados manifestaron que, pese a que la visibilidad del problema es mayor, todavía dista de ser considerado como problema de salud pública por quienes toman decisiones, como ilustra esta declaración recabada en una entrevista: "Hasta ahora, cuando menos, no existe una política pública, recientemente $[\ldots]$ casi todos los esfuerzos son a través de ONG o de fundaciones, como la LALCEC, como FUCA, que hacen atención a pacientes, se llevan a cabo conferencias, pero no hay una política pública hasta ahora".

\section{Legitimación de la política a través de la acción normativa}

En todos los países incluidos en el estudio se comprobó que las autoridades gubernamentales de salud han elaborado y habilitado mecanismos jurídiconormativos (que incluyen leyes de salud, reglamentos, planes y programas) para la detección temprana y la atención integral del cáncer de mama. Sus principales características se presentan en el cuadro 5 .

Los elementos más relevantes encontrados en los programas para la atención y el control del cáncer de mama son los siguientes. Primero, realización de mamografía. Pese a que en todos los países existe un programa oportunista 
CUADRO 5. Desarrollo jurídico-normativo en el cáncer de mama en cinco países de América Latina, 2010

\begin{tabular}{cl}
\hline País & \multicolumn{1}{c}{ Características } \\
\hline México & El programa es resultado de un proceso de planificación estratégica que definió objetivos, \\
& metas y líneas estratégicas, y un modelo de atención que incluye los ámbitos comunitario \\
& e individual. Su aplicación es de obligatorio cumplimiento para todo el sector salud. Lo más \\
& representativo en este aspecto son:
\end{tabular}

La Norma Técnica para la atención del cáncer de mama (NOM-041-SSA2-2011) formulada en el año 2002 y actualizada recientemente (publicación diario oficial 2011), y

el Programa Específico de Acción desarrollado desde el año 2001 y actualizado en 2007.

Brasil La acción normativa tiene como propósitos fundamentales: la asignación de recursos a las instituciones de salud; el entrenamiento al personal de salud dedicado a la detección y tratamiento del Cáncer de mama; la educación en el diagnóstico temprano a las mujeres en edades entre los 30 y los 50 años; la mejora de la estructura médica y la participación del sector privado y de las organizaciones sociales en el diseño de la política. Las normas específicas son:

Política Nacional de Oncología. PT no 2439 / GM de 8 de diciembre de 2005.

Política Nacional de Atención Integral a la Salud de la Mujer (PNAISM) 198.4.

El programa se denomina "Viva Mulher" 1998.

Venezuela Cuenta con un subprograma para el control del cáncer de mama, adscrito al Programa de Control de Cáncer (Ministerio del Poder Popular para la Salud), del cual despende el Registro Central de Cáncer.

Colombia La acción normativa específica se desarrolla a partir de 1993 con la inclusión de acciones específicas en los Planes de Beneficio (Plan Obligatorio de Salud-POS-) del Sistema de Seguridad Social en Salud: Resolución 5261 de 1994 Manual de Procedimientos e Intervenciones del POS. Más adelante se reglamenta el Plan Básico de Atención (acción gubernamental para toda la población) con la Norma técnica para la detección del cáncer de seno/Resolución 0412 de 2000. De reciente diseño y promulgación es la Ley 1384 de 2010 en la cual se establecen las acciones para la atención integral del cáncer.

Argentina El papel del Gobierno Nacional es asistir a las políticas provinciales a través de subsidios, recomendaciones y pautas, pero no tiene injerencia directa sobre las decisiones que toma cada una de ellas:

Resolución Secretarial № 2886/76. Programa Nacional de Control de Cáncer.

Resolución ministerial №59/03. Sub-programa de prevención secundaria del cáncer de mama.

Resolución 939/2000 del Ministerio de Salud. Programa Médico-Obligatorio (PMO).

Programa de Detección Precoz de Cáncer Genito-Mamario (PROGEMA).

brecha existente entre los sistemas de información de los países, un aspecto crítico para llevar a cabo la definición del problema. Brasil y México han logrado desarrollar los mejores sistemas de recolección de información de manera sistemática y habitual a escala nacional. De hecho, en Brasil este sistema ha adquirido un grado de sofisticación y de detalle importante, tanto desde el punto de vista epidemiológico (registro de la morbimortalidad) como administrativo (registros vinculados con las transiciones de las mujeres por los distintos niveles y dependencias de los servicios de salud). En México, este sistema se encuentra en pleno desarrollo y actualización desde 2007. Colombia, Argentina y Venezuela, por su parte, tienen sistemas de información segmentados (por entidad departamental, estatal o provincial) y con diferentes grados de desagregación, lo que impide tener información nacional para la toma de decisiones.

Con relación al posicionamiento del tema en la agenda política, se observó que se ha desarrollado de manera incremental en la Región. Se destaca como principal agente originador de las políticas (36) a las organizaciones de la sociedad civil de Brasil, Colombia y México. En estos mismos países se ha comprobado la participación incluyente en la toma de decisiones de instituciones gubernamentales y no gubernamentales, mientras que en Venezuela y Argentina la fuerte influencia proviene del sector gubernamental. Por tanto, esta participación todavía dista de ser un proceso de alcance nacional con características incluyentes para todos los sectores que deberían participar activamente. El grado de descentralización gubernamental y, por tanto, el de autonomía provincial, estatal o municipal podrían actuar como un factor explicativo de la dificultad para desarrollar este proceso, sobre todo en lo relacionado con la forma de desarrollarse la política o el programa. Otro factor que debe tenerse en cuenta es la forma como se transfieren los recursos financieros desde el nivel central, para garantizar la equidad regional necesaria para la implantación de la política o el programa.

El proceso de legitimación de la politica a través de la acción normativa es el que más ha avanzado en la Región. Se dispone de una extensa gama de marcos normativos y regulatorios amplios e incluyentes (relativos a la población y a las acciones para el control de la enfermedad), así como de guías y protocolos de manejo clínico consensuados entre las autoridades de salud, gremios académicos y científicos y organizaciones de la sociedad civil en todos los países, lo cual es un hallazgo que coincide con el trabajo realizado por González-Robledo, et al. 2009 (37). El hecho de que esta fase o etapa sea la más desarrollada en todos los países significa que los avances en las etapas de la formulación de las políticas no se producen necesariamente de forma lineal, resultado que corrobora lo señalado por Olavarría, et al. en 2011 (14).

Por otra parte, los resultados obtenidos en este estudio concuerdan con algunos hallazgos de Bridges, et al. en 2011 (38), en los cuales se identificaron temas comunes exitosos entre regiones (América Latina, Asia y Norte de África) en relación con el desarrollo de estrategias de control del cáncer de mama en países de recursos bajos y medios. Entre ellos merecen subrayarse la capacidad de construir escenarios participativos en las etapas de formulación de la política y la capacidad y experiencia de los profesionales locales, los responsables políticos y los líderes sociales.

Los retos a los que se enfrenta la Región para desarrollar este tema son fundamentalmente cuatro. El primero es articular la participación de las distintas instituciones, es decir, lograr acciones coordinadas entre los sectores gubernamental y no gubernamental. El segundo es conseguir una adecuada financiación de la política pública. El tercero es desarrollar sistemas de información que permitan tomar decisiones basadas en pruebas científicas. Este reto debe ser entendido en dos ámbitos: uno intranacional, que corresponde a cada país, y otro 
regional, que permita generar apoyo entre países. El cuarto y último es generar espacios de participación ordenada y sinérgica con la sociedad civil.

Para futuras investigaciones se recomienda reunir una gama más amplia de experiencias, que incluyan la visión de las personas afectadas por la enfermedad (pacientes).

Agradecimientos. Los autores agradecen el apoyo financiero y logístico de la American Cancer Society para la realización de este estudio y en particular a Syl- vana Rochet-Belleri, Alessandra Durstine y Cristina Parsons por su constante apoyo. Asimismo, agradecen a Rosa María Bejarano Arias su ayuda en la recolección de información y apoyo logístico del proyecto.

\section{Conflictos de interés. Ninguno.}

\section{REFERENCIAS}

1. Knaul FM, Arreola-Ornelas H, Lozano R, Gómez-Dantés H. Numeralia de Cáncer de Mama, marzo 2011. México, D.F.: Fundación Mexicana para la salud, Tómatelo a pecho; 2011. Disponible en: http://www.uv.mx/ noticias/cancerdemama.pdf Acceso el 25 de enero de 2012.

2. Bloom D. Breakaway: The global burden of cancer -challenges and opportunities. A report from the Economist Intelligence Unit Limited 2009. London: The Economist; 2009. Disponible en: http://livestrongblog.org/ GlobalEconomicImpact.pdf Acceso el 12 de julio de 2011.

3. Ferlay J, Shin HR, Bray F, Forman D, Mathers C, Parkin DM. Estimates of worldwide burden of cancer in 2008: GLOBOCAN 2008. Int J Cancer. 2010;127(12):2893-917.

4. Nigenda G, González-Robledo MC, González-Robledo LM, Bejarano RM. Políticas y programas gubernamentales de atención al cáncer de mama en América-Latina: México, Colombia, Venezuela, Brasil y Argentina. Work Paper. Cuernavaca: Instituto Nacional de Salud Pública, American Cancer Society: 2011.

5. Comisión Económica para América Latina y el Caribe. América Latina y el Caribe: Envejecimiento de la Población 1950-2050. Boletín Demográfico. 2003;No. 72; 39-74. Disponible en: http://www.sld.cu/galerias/ $\mathrm{pdf} /$ sitios/gericuba/america_latina_y_ el_caribe_el_envejecimiento_de_la_poblacion._1950-2050_cepal.pdf Acceso el 14 de agosto de 2010.

6. López-Ríos O, Lazcano-Ponce EC, TovarGuzmán V, Hernández Ávila M. La epidemiología del cáncer de mama en México. ¿Consecuencia de la transición demográfica? Salud Publica Mex. 1997;39(4):259-65.

7. Balducci L, Ershler WB. Cancer and aging: a nexus at several levels. Nat Rev Cancer. 2005;5(8):655-62.

8. Brown ML, Yabroff KR. Economic impact of cancer in the United States. En: Schottenfeld D, Fraumeni JF, eds. Cancer epidemiology and prevention. 3rd ed. New York: Oxford University Press; 2006.

9. Viñes J. La efectividad de la detección precoz de las enfermedades. Anales Sis San Navarra. 2007; 30(1):11-27. Disponible en: http:// www.cfnavarra.es/salud/anales/textos/ vol30/n1/colaba.html Acceso el 28 de septiembre de 2010.

10. International Agency for Research on Cancer, Working Group on the Evaluation of Cancer
Preventive Strategies. Efficacy of screening by breast self examination. En: Vaionio $\mathrm{H}$, Bianchini F, eds. Breast cancer screening. Lyon: IARC Press; 2002:107-13.

11. Anderson BO, Braun $S$, Lim $S$, Smith RA, Taplin S, Thomas DB. Early detection of breast cancer in countries with limited resources. Breast J. 2003; 9(Suppl2):S51-9.

12. Beláustegui, V. Conceptos básicos para la formulación de políticas de producción limpia. Montevideo: Comisión Económica para América Latina y el Caribe; 2007. Disponible en: http://www.eclac.cl/dmaah/noticias / paginas/8/28248/belaustegui.pdf Acceso el 14 de julio de 2011.

13. Velasco M. Distintos instrumentos para un mismo fin. Los instrumentos de las políticas públicas como herramienta para el análisis. Ponencia presentada en el VIII Congreso español de ciencia política y de la administración política para un mundo en cambio, Universidad Complutense, Valencia, España, del 18 al 20 de septiembre de 2007. Disponible en: http://eprints.ucm.es/12184/2/ velasco_Instrumentos_pol\%C3\%ADticas. pdf Acceso el 26 de agosto de 2010.

14. Olavarría-Gambi M, Navarrete-Yáñez B, Figueroa-Huencho V. ¿Cómo se formulan las políticas públicas en Chile? Evidencia desde un estudio de caso. Rev Pol Gob. 2011;18(1):109-54.

15. Hogwood B, Gunn L. Policy analysis for the real world. J Soc Pol. 1986;15(1):132-3.

16 Dye Thomas R. Understanding public policy. 9th edition. Upper Saddle River: Prentice Hall; 1998.

17. Doreian P, Woodard KL. Defining and locating cores and boundaries of social networks. Soc Networks. 1994;16(4):267-93.

18. Bertaux, D. Los relatos de vida en el análisis social. En Aceves J (Compilador). Historia y fuente oral. México, D.F.: Instituto de Investigaciones "José Mará Luis Mora”. 1989;134-48.

19. Public policy and public participation: Engaging citizens and community in the development of public policy. Produced by Bruce L. Smith for the Population and Public Health Branch, Halifax: Atlantic Regional Office, Health Canada; 2003. Disponible en: http:// www.phac-aspc.gc.ca/canada/regions/ atlantic/pdf/pub_policy_partic_e.pdf Acceso el 5 de mayo de 2011.

20. Glaser BG, Strauss AL. The discovery of grounded theory. Strategies for qualitative research. New York: Aldine Publishing Company; 1967.
21. Murphy E, Dingwall R, Greatbatch D, Parker S, Watson P. Qualitative research methods in health technology assessment: a review of the literature. Health Technol Assess. 1998;2(16):1-274.

22. Greene JC. Is mixed methods social inquiry a distinctive methodology? J Mix Meth Res. 2008; 2(1):7-22.

23. Asociación Médica Mundial. Principios éticos para las investigaciones médicas en seres humanos: Declaración de Helsinki de la Asociación Médica Mundial. Helsinki: Asociación Médica Mundial; 1964. Disponible en: www.fisterra.com/mbe/investiga/declaracion_helsinki.asp Acceso el 19 de octubre de 2010.

24. Schwan R, Gildeza C, Santana M, Lagarrigue Z, Schwan E. Programa provincial de prevención secundaria del cáncer de mama, Tucumán 2002-2011, y para detección precoz del cáncer de mama. Rev Facultad Med. 2006;7(1):48-51.

25. Viniegra M, Paolino M, Arrossi S. Cáncer de mama en Argentina: organización, cobertura y calidad de las acciones de prevención y control. Informe final Julio 2010: diagnóstico de situación del Programa nacional y programas provinciales. Primera ed. Buenos Aires: Organización Panamericana de la Salud; 2010. Disponible en: http://www.msal.gov.ar/inc/ descargas/cancer_de_mama.pdf Acceso el 15 de enero de 2011.

26. Bodstein R, coord. História e Saúde Pública: a política de controle do cáncer no Brasil. Rio de Janeiro: Escola Nacional de Saúde Pública; 1987.

27. Instituto Nacional de Câncer. Estimativa 2010: incidência de câncer no Brasil. Rio de Janeiro: Instituto Nacional de Câncer; 2009.

28. Capote Negrín LG. Aspectos epidemiológicos del cáncer en Venezuela. Rev Venez Oncol. 2006;18(4):269-81. Disponible en: http:// www.scielo.org.ve/scielo.php?pid=S0798 05822006000400011\&script=sci_arttext Acceso el 15 de diciembre de 2010.

29. López N. 10 años de experiencia del Programa de cáncer de cuello uterino y mama, Sistema de Información en Salud. Ponencia realizada en la I Jornada de Epidemiología del Cáncer, CORPOSALUD. Aragua, Venezuela, julio de 2010. Disponible en: http://www. iaes.edu.ve/images/noticias2010/julio/ Nora_Lopez.pdf Acceso el 17 de diciembre de 2010.

30. Piñeros $M$, Hernández $G$, Bray $F$. Increasing mortality rate of common malignancies 
in Colombia: an emerging problem. Cancer. 2004;101(10):2285-92.

31. Martínez-Bejarano R, Martínez-Salgado, C. La mortalidad por cáncer cervicouterino y de mama en Colombia y México como expresión de las desigualdades socioeconómicas y de género. Trabajo presentado en el III Congreso de la Asociación Latinoamericana de Población, Córdoba, Argentina, del 24 al 26 de septiembre de 2008. Disponible en: http://www.alapop.org/2009/images/ DOCSFINAIS_PDF/ALAP_2008 FINAL_155.pdf Acceso el 13 de octubre de 2010.

32. República de Colombia. Ley 1384 de 2010. Disponible en: http://www.secretariasenado.gov.co/senado/basedoc/ley/2010/ ley_1384_2010.html Acceso el 12 de octubre de 2010 .
33. Knaul F, Lozano R, Arreola H, Gómez Dantes H, México: Numeralia de Cáncer de Mama, 2008. México, D.F.: Fundación Mexicana para la Salud; 2008. Disponible en: http://www. salud.carso.org/docum/hoja_\%20datos_ cama.pdf Acceso el 25 de agosto de 2011.

34. López-Carrillo L, Suárez-López L y TorresSánchez L. Detección del cáncer de mama en México: síntesis de los resultados de la Encuesta Nacional de Salud Reproductiva. Salud Publica Mex. 2009;51(Supl. 2): S335-49.

35. Secretaría de Salud. Programa de Acción 2007-2012. Cáncer de Mama. $1^{a}$ ed. México, D.F.: Secretaría de salud; 2008.

36. Ruelas-Barajas E, Gay-Molina JG. Políticas públicas, estructuras del Estado y defensa del derecho a la protección de la salud. Salud Pública Mex. 2008;50(Supl 3):S343-7.
37. González-Robledo LM, González-Robledo MC, Nigenda G, López-Carrillo L. Acciones gubernamentales para la detección temprana del cáncer de mama en América Latina. Retos a futuro. Salud Publica Mex. 2010;52(6): 533-43.

38. Bridges J, Anderson BO, Buzaid AC, Jazieh AJ, Niessen LW, Blauvelt B, et al. Identifying important breast cancer control strategies in Asia, Latin America and the Middle East/ North Africa. Health Serv Res. 2011;11:227. doi:10.1186/1472-6963-11-227. Disponible en: http:/ / www.biomedcentral.com/ 1472-6963/11/227 Acceso el 4 de mayo de 2012.

Manuscrito recibido el 13 de abril de 2012. Aceptado para publicación, tras revisión, el 18 de diciembre de 2012.

ABSTRACT Objective. To understand the public policy-making process as it relates to breast cancer care in five Latin American countries.

\section{Public policy-making on breast cancer in Latin America}

Methods. An exploratory-evaluative study was conducted in Argentina, Brazil, Colombia, Mexico, and Venezuela in 2010, with the selection of countries based on convenience sampling. Sixty-five semi-structured interviews were conducted with government officials, academics, and representatives of trade associations and civil society organizations. A content analysis of secondary sources was performed. Information sources, data, and informants were mixed using the triangulation method for purposes of analysis.

Results. The countries that have made the most progress in public policy-making related to breast cancer are Brazil and Mexico. Although Argentina, Colombia, and Venezuela do not have policies, they do have breast cancer care programs and activities. Two perspectives on the development of public policies became evident: the first includes the broad participation of both governmental and nongovernmental sectors, whereas the second, more narrow approach involves government authorities alone.

Conclusions. The results point to significant differences in public policy-making related to breast cancer in the Region. They also show that greater progress has been made in countries where policies have been developed through inclusive participation processes.

Key words Health public policy; breast neoplasms; Latin America. 